Miguel Calero Rojas, Aurora Jurado Roger*, Marta Gutiérrez Grúa, Lourdes de la Peña Carretero, Victoria Romero Sotomayor, Javier López Braos, Federico Izquierdo Carrasco, Luis Herrero Tabanera y Carmen Moreno Aguilar

\title{
Mejora del protocolo de cribado de diabetes gestacional: estudio de validación diagnóstica
}

https://doi.org/10.1515/almed-2020-0118 Recibido 22-07-2020; aceptado 12-11-2020; publicado en línea 11-02-2021

\section{Resumen}

Objetivos: El objetivo de este trabajo es evaluar la validez diagnóstica de dos métodos de cribado de diabetes mellitus gestacional (DMG).

Métodos: Se realizó un estudio prospectivo de validación diagnóstica en 2007 embarazadas. Las participantes fueron asignadas al grupo de alto o bajo riesgo, dependiendo de los factores de riesgo que presentaran. Se realizó una prueba de $\mathrm{HbA}_{1 \mathrm{c}}$ simultáneamente al protocolo estándar basado en el test de O'Sullivan (TOS). Posteriormente, se aplicó un algoritmo que combinaba los resultados de ambos biomarcadores.

Resultados: En el grupo de bajo riesgo, el TOS mostró un área bajo la curva mayor (AUC 0,953; IC95\% 0,915-0,992) que la prueba de $\mathrm{HbA}_{1 \mathrm{c}}(0,688$; IC $95 \% 0,541-0,834)$. El mejor punto de corte del TOS, $153,5 \mathrm{mg} / \mathrm{dL}(8,52 \mathrm{mmol} / \mathrm{L})$, mostró mayor validez diagnóstica que el de la $\mathrm{HbA}_{1 c}$, $28 \mathrm{mmol} / \mathrm{mol}(4,75 \%)$ y que el algoritmo basado en ambas pruebas. En el grupo de alto riesgo, el TOS mostró mejor rendimiento diagnóstico que la $\mathrm{HbA}_{1 \mathrm{c}}$ y el algoritmo. Los puntos de corte óptimos para el TOS fueron mayores que los recomendados en los protocolos actuales. Semana 13:

\footnotetext{
*Autora para correspondencia: Aurora Jurado Roger, MD, PhD, Departamento de Inmunología y Alergia, Hospital Universitario Reina Sofía, Avda. Menéndez Pidal s/n, 14004, Córdoba, España; and Immunlogy and Allergy Unit, Hospital Universitario Reina SofíaIMIBIC, Córdoba, España, Phone: 34 + 957011 490,

E-mail: aurora.jurado.sspa@juntadeandalucia.es

Miguel Calero Rojas, Marta Gutiérrez Grúa and Federico Izquierdo Carrasco, Obstetrics and Gynaecology Unit, Hospital Infanta Margarita, Cabra, España

Lourdes de la Peña Carretero, Victoria Romero Sotomayor and Javier López Braos, Laboratory Unit, Hospital Infanta Margarita, Cabra, España

Luis Herrero Tabanera, Operation Control Unit, Hospital Infanta Margarita, Cabra, España

Carmen Moreno Aguilar, Immunlogy and Allergy Unit, Hospital Universitario Reina Sofía- IMIBIC, Córdoba, España
}

TOS AUC 0,882 (IC 95\% 0,843-0,921), $\mathrm{HbA}_{1 \mathrm{c}}$ AUC 0,624 (IC $95 \%$ 0,562-0,686), punto de corte para el TOS $140,5 \mathrm{mg} / \mathrm{dL}$ $(7,8 \mathrm{mmol} / \mathrm{L})$, punto de corte para la $\mathrm{HbA}_{1 \mathrm{c}} 33 \mathrm{mmol} / \mathrm{mol}$ (5,15\%). Semana 24: TOS AUC 0,944 (IC 95\% 0,925-0,962), $\mathrm{HbA}_{1 \mathrm{c}}$ AUC 0,642 (IC 95\% 0,575-0,709), punto de corte para el TOS, $145,5 \mathrm{mg} / \mathrm{dL}(8,08 \mathrm{mmol} / \mathrm{L})$, punto de corte para la $\mathrm{HbA}_{1 \mathrm{c}} 29 \mathrm{mmol} / \mathrm{mol}(4,85 \%)$.

Conclusiones: El método para el cribado de DMG basado en el test de O'Sullivan con valores límite superiores a los recomendados fue el que mostró mejor validez diagnóstica. Si se hubieran aplicado estos umbrales, se habría evitado la prueba de sobrecarga oral de glucosa con $100 \mathrm{~g}$ al 55,6\% y al 13,7\% de las gestantes de bajo y alto riesgo.

Palabras clave: eventos adversos; diabetes gestacional; test de O'Sullivan; hemoglobina glIcosilada; cribado.

\section{Introducción}

La diabetes mellitus gestacional (DMG) se ha definido tradicionalmente como cualquier grado de intolerancia a la glucosa que aparece o es identificada por primera vez durante el embarazo [1]. Esta definición ha sido modificada recientemente por la Organización Mundial de la Salud [2] y la Asociación Americana de Diabetes [3], pasando a aplicar el término DMG a aquellos casos diagnosticados en el segundo o tercer trimestre de embarazo, que no sean diabetes de tipo 1 o 2. Sin embargo, estos nuevos criterios no han sido aceptados universalmente, y la definición y el diagnóstico de la misma sigue siendo objeto de un intenso debate [4-7].

De acuerdo con la definición clásica de DMG, su prevalencia oscila entre el 2\% y el 17\% [7, 8]. En España, la probabilidad de desarrollar DMG es del 8,8\% [9].

El Grupo Español de Diabetes y Embarazo (GEDE) clasifica a las embarazadas en dos grupos: alto riesgo, y riesgo bajo/moderado. La DMG está relacionada con los siguientes factores de riesgo: edad ( $>35$ años), obesidad (IMC $>30 \mathrm{~kg} / \mathrm{m}^{2}$ ), antecedentes de DMG, familiar de primer grado con DM, y antecedentes de macrosomía [10]. 
El método validado en España para el diagnóstico de DMG consta de dos pasos: el cribado con el test de O'Sullivan (TOS) y la prueba de sobrecarga oral de glucosa (SOG). El TOS se realiza en la semana $24^{\mathrm{a}}$ de gestación (semana $13^{\mathrm{a}}$, si existe algún factor de riesgo). Si el TOS es positivo ( $\geq 140 \mathrm{mg} / \mathrm{dL} ; \geq 7,77 \mathrm{mmol} / \mathrm{L}$ ), se realiza la SOG. El TOS consiste en la administración oral de $50 \mathrm{~g}$ de glucosa y la medición de glucemia al cabo de una hora. La SOG consiste en la administración de $100 \mathrm{~g}$ de glucosa, que se mide antes de la administración y transcurridas una, dos y tres horas [10, 11]. Las gestantes suelen mostrar mala tolerancia a estas pruebas, que suelen provocarles vómitos e impiden que la prueba llegue a término. Otras limitaciones de estos métodos son la duración y la glucólisis preanalítica en muestras de glucosa en plasma, que pueden llevar al infradiagnóstico de la DMG [4, 6, 12-16].

En 2009, el Comité Internacional de Expertos en Diabetes recomendó la prueba de la hemoglobina A glicosilada $\left(\mathrm{HbA}_{1 \mathrm{c}}\right)$ como la prueba de elección para el manejo crónico de la diabetes [16]. La prueba de la $\mathrm{HbA}_{1 \mathrm{c}}$ ofrece varias ventajas frente a las pruebas basadas en los niveles de glucosa en plasma, como son la estandarización, una mejor correlación con la incidencia de eventos adversos a largo plazo, una menor incidencia de errores preanalíticos, no precisar la toma secuencial de varias muestras y un menor impacto causado por las perturbaciones agudas en los niveles de glucosa. No obstante, la precisión diagnóstica de la $\mathrm{HbA}_{1 \mathrm{c}}$ en el diagnóstico de la diabetes durante el embarazo, un periodo en el que se producen cambios en el recambio de eritrocitos, podría disminuir, por lo que se recomienda realizar pruebas basadas en la medición de glucosa $[7,17]$.

Diversos artículos han demostrado que la prueba de la $\mathrm{HbA}_{1 \mathrm{c}}$ podría ser útil en el diagnóstico de DM en individuos con alto riesgo $[18,19]$. Sin embargo, aún no se han realizado suficientes estudios que validen el uso de la $\mathrm{HbA}_{1 \mathrm{c}}$ en el diagnóstico de la DMG [20-24] y su utilidad sigue siendo objeto de controversia [25].

De este modo, el propósito de este estudio es evaluar la validez diagnóstica de dos nuevas estrategias, que causen menos molestias a las embarazadas, para el cribado de DMG.

\section{Materiales y métodos}

Este estudio prospectivo se realizó en un hospital terciario (Hospital Infanta Margarita). Se incluyó a todas las embarazadas tratadas en el hospital y en su área de referencia (Sur de Córdoba) durante un periodo de tres años. La investigación se realizó de conformidad con la legislación nacional, las políticas institucionales, y de acuerdo con los principios de la Declaración de Helsinki. Así mismo, este estudio fue aprobado por el Comité de Ética del Hospital Reina Sofía, Córdoba (España). Este estudio fue financiado por una ayuda del Instituto Nacional de Salud Carlos III (ISCIII) (PI11 01064).

Asumiendo una prevalencia del 10\%, una sensibilidad del 85\%, una especifidad del $85 \%$, y un porcentaje permitido de errores de tipo II del 5\%, con un nivel de significación del $95 \%$, con el programa Epidat 4.1 (Conselleria de Sanidade Galega) se calculó una muestra de 1.970 participantes.

Se incluyó a todas las gestantes atendidas en la Unidad de Obstetricia entre septiembre de 2011 y septiembre de 2014 que aceptaron participar en el estudio. Todas las participantes firmaron un consentimiento informado. Se excluyó a las mujeres con diabetes pregestacional, hemoglobinopatías o cualquier situación asociada con un mayor recambio de glóbulos rojos (anemia, transfusión).

La muestra inicial estaba compuesta por 2.270 mujeres. Se excluyó a 221 por diversas razones: pérdida durante el seguimiento (134), aborto espontáneo (39), seguimiento en un centro privado (31) y datos demográficos incompletos (17). Además, se excluyó del análisis final a 42 participantes, debido a que recibieron una transfusión (35), presentaron diabetes pregestacional (3), hemoglobinopatías (2) o abandonaron el estudio. La muestra final incluida en el análisis estadístico estuvo compuesta por un total de 2,007 mujeres.

A las participantes se les realizó un examen físico y se les entregó un cuestionario para identificar factores de riesgo de DMG: edad ( $>35$ años), obesidad (IMC $>30 \mathrm{~kg} / \mathrm{m}^{2}$ ), antecedentes de DMG, familiar de primer con DM, antecedentes de macrosomía y origen étnico con elevada prevalencia de DMG (africanas, latinoamericanas o asiáticas).

Se realizó el TOS y una prueba de $\mathrm{HbA}_{1 \mathrm{c}}$ entre la semana $24^{\mathrm{a}}$ y la semana $26^{\mathrm{a}}$ de embarazo (GCT-s24; $\mathrm{HbA}_{1 \mathrm{c}}$-s24). Si los resultados del TOS eran positivos ( $\geq 140 \mathrm{mg} / \mathrm{dL} ; \geq 7,77 \mathrm{mmol} / \mathrm{L})$, se realizó la SOG con 100 g. Una SOG se consideró positiva si $\geq 105 \mathrm{mg} / \mathrm{dL}(\geq 5,83 \mathrm{mmol} / \mathrm{L})$ (basal), $\geq 190 \mathrm{mg} / \mathrm{dL}$ ( $\geq 10,55 \mathrm{mmol} / \mathrm{L}$ ) $(1 \mathrm{~h}), \geq 165 \mathrm{mg} / \mathrm{dL}$ ( $\geq 9,16 \mathrm{mmol} / \mathrm{L})$ ( $2 \mathrm{~h}$ ), $\geq 145 \mathrm{mg} / \mathrm{dL}$ ( $\geq 8,05 \mathrm{mmol} / \mathrm{L}$ ) (3 h) [6, 7]. El diagnóstico de DMG se confirmó si se cumplía alguno de los siguientes criterios: nivel de glucosa en plasma $\geq 126 \mathrm{mg} / \mathrm{dL}$ ( $\geq 6,99 \mathrm{mmol} / \mathrm{L}$ ) (medido dos veces), glucemia aleatoria $\geq 200 \mathrm{mg} / \mathrm{dL}$ ( $\geq 11,1 \mathrm{mg} / \mathrm{L}$ ) y dos o más puntos alterados en la SOG (según los criterios del GEDE [10] y del Grupo Nacional de Datos sobre la Diabetes [11]). Las mujeres que presentaron factores de riesgo de DMG fueron evaluadas en dos ocasiones siguiendo el mismo esquema: en la semana $13^{\mathrm{a}}$ de gestación (GCT-s13; $\mathrm{HbA}_{1 \mathrm{c}}$-s13) y entre la semana $24^{\mathrm{a}}$ y $26^{\mathrm{a}}$

Dependiendo de la epidemiología, historia clínica y los resultados analíticos, los obstetras, que desconocían los resultados de la $\mathrm{HbA}_{1 \mathrm{c}}$ asignaron a las participantes a diferentes grupos.

$\mathrm{El}$ análisis clínico de las pruebas de glucosa y de $\mathrm{HbA}_{1 \mathrm{c}}$ fue realizado por diferentes técnicos de laboratorio y diferentes facultativos, que desconocían respectivamente el resultado de la prueba alternativa, así como el grupo al que pertenecían las participantes.

Procedimientos analíticos (véase el Material Suplementario).

Análisis estadístico (véase el Material Suplementario).

Estrategias para el cribado de la DMG (véase Material Suplementario).

\section{Resultados}

La distribución de frecuencias de las variables continuas no presentó una distribución normal en ninguna de las 
cohortes (población total, gestantes con o sin factores de riesgo). En la Tabla 1 se detallan los resultados del análisis descriptivo.

\section{Análisis de la población}

La prevalencia de la DMG en toda la población fue del $5,7 \%$. Las medianas de las variables edad, TOS-s2 y $\mathrm{HbA}_{1 c^{-}}$ s24 fueron significativamente superiores en las embarazadas con factores de riesgo $(\mathrm{p}<0,001)$. Las participantes que desarrollaron DMG tenían mayor edad y presentaban niveles superiores de $\mathrm{TOS}$ y $\mathrm{HbA}_{1 \mathrm{c}}(\mathrm{p}<0,001)$ en la semana $24^{\mathrm{a}}$. La presencia de factores de riesgo, especialmente la edad superior a los 35 años, un IMC $>30 \mathrm{~kg} / \mathrm{m}^{2}$, antecedentes de DMG y antecedentes familiares de DM fueron significativamente más frecuentes en aquellas participantes que desarrollaron DMG, frente a las que no lo hicieron $(\mathrm{p}<0,001)$ (Tablas 2 y 3 ). Aunque 79 participantes habían nacido en otro país (20 nacionalidades distintas), solo dos de ellas no eran caucásicas.

La regresión logística no mostró un buen rendimiento diagnóstico, ya que, aunque el análisis clasificó correctamente al $96,9 \%$ de las participantes, solo identificó correctamente el 37,7\% de los casos de DMG.
En términos de precisión diagnóstica, el TOS mostró una mayor AUC que la $\mathrm{HbA}_{1 c}$; $(0,953$ vs. 0,672 , respectivamente) (Figura 1A; Tabla 4). El mejor punto de corte para TOS-s24 fue $145,5 \mathrm{mg} / \mathrm{dL}(8,08 \mathrm{mmol} / \mathrm{L})$ (Sensibilidad: 95,1\%; Especifidad: 85,7\%; VPP: 22,19\%; VPN: 99,75\%). Para $\mathrm{HbA}_{1 \mathrm{c}}$-s24, el mejor punto de corte fue $29 \mathrm{mmol} / \mathrm{mol}$ (4,85\%) (Sensibilidad: 67\%; Especifidad: 57,8\%; VPP: 7,6\%; VPN: 97,12\%) (Tabla 4).

\section{Análisis de la población sin factores de riesgo}

Un total de 1,054 participantes no presentaron factores de riesgo. La prevalencia de la DMG en esta población fue del $1,8 \%$. Los valores de TOS y $\mathrm{HbA}_{1 \mathrm{c}}$ en la semana $24^{\mathrm{a}}$ fueron significativamente más elevados en las participantes que desarrollaron DMG. No se hallaron diferencias en términos de edad (Tabla 2).

La regresión logística no mostró un buen rendimiento diagnóstico, ya que, aunque el análisis clasificó correctamente al $98,4 \%$ de las participantes, solo identificó correctamente el 22\% de los casos de DMG.

En cuanto a la precisión diagnóstica, el TOS mostró una mayor AUC que la $\mathrm{HbA}_{1 c}(0,953$ frente a 0,688 ,

Tabla 1: Distribución de las variables clínicas de la población del estudio.

\begin{tabular}{|c|c|c|c|}
\hline Variables clínicas & $\begin{array}{l}\text { Todas las gestantes } \\
(\mathrm{n}=2007) \\
\text { Mediana }\left(\mathrm{IR}^{\mathrm{a}}\right)\end{array}$ & $\begin{array}{l}\text { Grupo de bajo riesgo } \\
(n=1054) \\
\text { Mediana (RI) }\end{array}$ & $\begin{array}{r}\text { Grupo de alto riesgo } \\
(\mathrm{n}=953) \\
\text { Mediana (RI) }\end{array}$ \\
\hline Edad, años & $31(28-35)$ & $29(26-32)$ & $34(31-37)$ \\
\hline TOS-s13 $3^{\mathrm{b}}, \mathrm{mg} / \mathrm{dL}$ & $n a^{c}$ & na & $109(89-131)$ \\
\hline TOS-s13, mmol/L & na & na & $6,05(4,94-7,27)$ \\
\hline $\mathrm{HbA}_{1 c^{-}} \mathrm{s} 13^{\mathrm{d}}, \mathrm{mmol} / \mathrm{mol}$ & na & na & $31,1(29-33,3)$ \\
\hline $\mathrm{HbA}_{1 c^{-}-\mathrm{s} 13, \%}$ & na & na & $5(4,8-5,2)$ \\
\hline TOS-s $24^{\mathrm{e}}, \mathrm{mg} / \mathrm{dL}$ & $115(96-138)$ & $110(93-132)$ & $122(102-144)$ \\
\hline TOS-s24, mmol/L & $6,38(5,33-7,66)$ & $6,11(5,16-7,33)$ & $6,77(5,66-7,99)$ \\
\hline $\mathrm{HbA}_{1 \mathrm{c}}-\mathrm{s} 24^{\mathrm{f}}, \mathrm{mmol} / \mathrm{mol}$ & $29(25,7-31,1)$ & $28(25,7-31,1)$ & $29(26,8-32,2)$ \\
\hline \multirow[t]{2}{*}{$\mathrm{HbA}_{1 \mathrm{c}}-\mathrm{s} 24, \%$} & $4,8(4,5-5)$ & $4,7(4,5-5)$ & $4,8(4,6-5,1)$ \\
\hline & $\%$ & $\%$ & $\%$ \\
\hline Prevalencia $\mathrm{DMG}^{\mathrm{g}}$ & 5,7 & 1,8 & 10 \\
\hline Factores de riesgo & 47,5 & 0 & 1 \\
\hline Edad $>35$ años & 22,5 & 0 & 47,4 \\
\hline $\mathrm{BMI}^{\mathrm{h}}>30 \mathrm{~kg} / \mathrm{m}^{2}$ & 13,7 & 0 & 28,8 \\
\hline Macrosomía & 2,2 & 0 & 4,7 \\
\hline$H P^{i}$ de $D G$ & 3,4 & 0 & 7,1 \\
\hline $\mathrm{HF}^{\mathrm{j}}$ de diabetes & 24,7 & 0 & 51,9 \\
\hline Origen étnico de riesgo & 0,1 & 0 & 0,2 \\
\hline
\end{tabular}

$\mathrm{RI}^{\mathrm{a}}$, rango intercuartílico; TOS-s13 $3^{\mathrm{b}}$, test de O'Sullivan en la semana $13^{\mathrm{a}} ; \mathrm{na}^{\mathrm{c}}$, No aplicable; $\mathrm{HbA}_{1 \mathrm{c}}-\mathrm{s} 13^{\mathrm{d}}$, prueba de hemoglobina glicosilada en la semana $13^{\mathrm{a}}$; TOS-s2 $4^{\mathrm{e}}$, test de O'Sullivan en la semana $24^{\mathrm{a}}$; $\mathrm{HbA}_{1 \mathrm{c}}-\mathrm{s} 24^{\mathrm{f}}$, prueba de hemoglobina glicosilada en la semana $24^{\mathrm{a}}$; DMG ${ }^{\mathrm{g}}$, Diabetes mellitus gestacional; IMCh , Índice de masa corporal; $\mathrm{HP}^{\mathrm{i}}$, Historia personal; $\mathrm{HF}^{\mathrm{j}}$, historia familiar. 
Tabla 2: Comparación de variables continuas entre las gestantes que desarrollaron (casos) y no desarrollaron (controles) DMG .

\begin{tabular}{|c|c|c|c|c|}
\hline Población & Variables continuas & Casos: mediana (RI') & Controles: mediana (RI) & VALOR p \\
\hline \multirow[t]{5}{*}{ Todas las gestantes } & Edad, años & $34(30,75-37)$ & $31(28-34)$ & $<0,001$ \\
\hline & TOS-s $24^{\mathrm{c}}, \mathrm{mg} / \mathrm{dL}$ & $168(156-187,5)$ & $114(96-135)$ & $<0,001$ \\
\hline & TOS-s24, mmol/L & $9,32(8,69-10,41)$ & $6,33(5,33-7,49)$ & \\
\hline & $\mathrm{HbA}_{1 \mathrm{c}}-\mathrm{s} 24^{\mathrm{d}}, \mathrm{mmol} / \mathrm{mol}$ & $31(28-36)$ & $29(26-31)$ & $<0,001$ \\
\hline & $\mathrm{HbA}_{1 \mathrm{c}}-\mathrm{s} 24, \%$ & $5(4,7-5,4)$ & $4,8(4,5-5)$ & \\
\hline \multirow[t]{5}{*}{ Gestantes sin factores de riesgo } & Edad, años & $30(28-33)$ & $29(26-32)$ & $\mathrm{ns}^{\mathrm{e}}$ \\
\hline & TOS-s24, mg/dL & $167(160-180)$ & $110(92-130)$ & $<0,001$ \\
\hline & TOS-s24, mmol/L & $9,27(8,88-9,99)$ & $6,11(5,11-7,22)$ & \\
\hline & $\mathrm{HbA}_{1 \mathrm{c}}-\mathrm{s} 24, \mathrm{mmol} / \mathrm{mol}$ & $31(29-37)$ & $28(26-31)$ & 0,006 \\
\hline & $\mathrm{HbA}_{1 \mathrm{c}}-\mathrm{s} 24, \%$ & $5(4,77-5,5)$ & $4,7(4,5-5)$ & \\
\hline \multirow[t]{9}{*}{ Gestantes con factores de riesgo } & Edad, años & $35(31-37)$ & $34(31-37)$ & ns \\
\hline & TOS-s13, mg/dL & $163(134,5-184)$ & $106(89-125)$ & $<0,001$ \\
\hline & TOS-s13, mmol/L & $9,05(7,46-10,21)$ & $5,88(4,77-6,94)$ & \\
\hline & $\mathrm{HbA}_{1 \mathrm{c}^{-}} \mathrm{s} 13^{\mathrm{g}}, \mathrm{mmol} / \mathrm{mol}$ & $33(30-36)$ & $31(29-33)$ & $<0,001$ \\
\hline & $\mathrm{HbA}_{1 \mathrm{c}}-\mathrm{s} 13, \%$ & $5,2(4,9-5,4)$ & $5(4,8-5,2)$ & \\
\hline & TOS-s24, mg/dL & $168(154,7-189,7)$ & $119,5(100-139)$ & $<0,001$ \\
\hline & TOS-s24, mmol/L & $9,32(6,59-10,53)$ & $6,63(5,55-7,71)$ & \\
\hline & $\mathrm{HbA}_{1 \mathrm{c}}-\mathrm{s} 24, \mathrm{mmol} / \mathrm{mol}$ & $31(28-34)$ & $29(27-32)$ & $<0,001$ \\
\hline & $\mathrm{HbA}_{1 \mathrm{c}}-\mathrm{s} 24, \%$ & $5(4,7-5,3)$ & $4,8(4,6-5,1)$ & \\
\hline
\end{tabular}

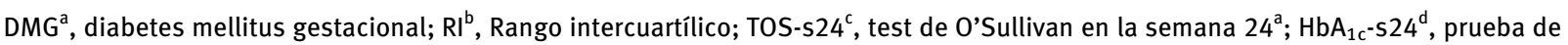
hemoglobina glicosilada en la semana $24^{\mathrm{a}}$; $\mathrm{ns}^{\mathrm{e}}$, no significativa; TOS-s13 $3^{\mathrm{f}}$, test de $0^{\prime}$ Sullivan en la semana $13^{\mathrm{a}} ; \mathrm{HbA}_{1 \mathrm{c}^{-}} \mathrm{S} 13^{\mathrm{g}}$, prueba de hemoglobina glicosilada en la semana $13^{\mathrm{a}}$.

Tabla 3: Odds ratios para cada factor de riesgo (variables discretas) para desarrollar $\mathrm{DMG}^{\mathrm{a}}$.

\begin{tabular}{|c|c|c|c|c|}
\hline Población & Variables discretas & $O R^{b}$ & $I^{C} 95 \%$ & Valor $p$ \\
\hline \multirow[t]{6}{*}{ Todas las gestantes } & Tener factores de riesgo & 6,03 & $3,65-9,95$ & $<0,001$ \\
\hline & Edad $>35$ years & 2,11 & $1,42-3,13$ & $<0,001$ \\
\hline & Macrosomía & & & $N s^{d}$ \\
\hline & $I M C^{e}>30 \mathrm{~kg} / \mathrm{m}^{2}$ & 3,83 & $2,54-5,76$ & $<0,001$ \\
\hline & HPf de DMG & 13,02 & $7,63-22,2$ & $<0,001$ \\
\hline & $\mathrm{HF}^{\mathrm{g}}$ de $\mathrm{DMG}$ & 2,44 & $1,66-3,59$ & $<0,001$ \\
\hline \multirow[t]{5}{*}{ Mujeres con factores de riesgo } & Edad $>35$ years & & & ns \\
\hline & Macrosomía & & & ns \\
\hline & $I M C>30 \mathrm{~kg} / \mathrm{m}^{2}$ & 1,93 & $1,25-2,99$ & $<0,001$ \\
\hline & HP de DMG & 7,32 & $4,23-12,65$ & $<0,001$ \\
\hline & HF de DMG & & & ns \\
\hline
\end{tabular}

$\mathrm{DMG}^{\mathrm{a}}$, diabetes mellitus gestacional; $\mathrm{OR}^{\mathrm{b}}$, Odds Ratio; $\mathrm{IC}^{\mathrm{c}}$, Intervalo de confianza; $\mathrm{ns}^{\mathrm{d}}$, no significativo; IMC ${ }^{\mathrm{e}}$, Índice de masa corporal; $\mathrm{HP}^{\mathrm{f}}$, History personal; $\mathrm{HF}^{\mathrm{g}}$, Historia familiar.

respectivamente) (Figura 1B). El mejor punto de corte para el TOS fue $153,5 \mathrm{mg} / \mathrm{dL}(8,52 \mathrm{mmol} / \mathrm{L})$ (Sensibilidad: $89,5 \%$; Especifidad: 93,6\%; VPP: 20,48\%; VPN: 99,79\%). Para la $\mathrm{HbA}_{1 \mathrm{c}}$, el mejor punto de corte fue de $28 \mathrm{mmol} / \mathrm{mol}(4,75 \%)$ (Sensibilidad: 77,8\%; Especifidad: 52,1\%; VPP: 2,87\%; VPN: 99,22\%) (Tabla 4). Además, se determinaron dos umbrales extremos de $\mathrm{HbA}_{1 \mathrm{c}}$ en esta población. El umbral de $\mathrm{HbA}_{1 \mathrm{c}}$ de $25 \mathrm{mmol} / \mathrm{mol}(4,45 \%)$ mostró una sensibilidad similar a la del TOS, por lo que se podría evitar la realización del TOS en las mujeres con valores inferiores a este umbral (sensibilidad 88,9\%). Un valor de $37 \mathrm{mmol} / \mathrm{mol}$ (5,55\%) mostró una especifidad de $98,6 \%$ a la hora de diagnosticar la DMG. 
A

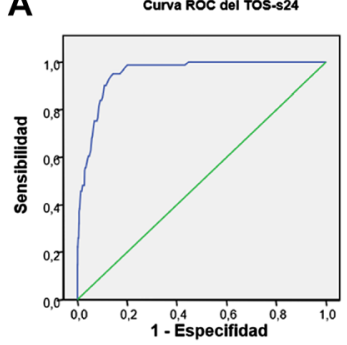

Punto de corte: $145,5 \mathrm{mg} / \mathrm{dL}$ ( $8,08 \mathrm{mmol} / \mathrm{L})$ Curva ROC del TOS-s24

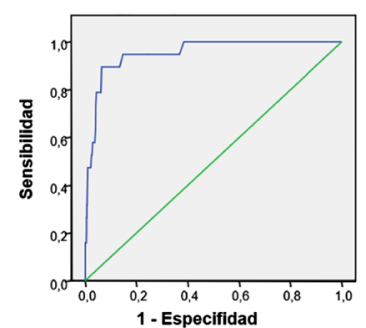

C Punto de corte: $153,5 \mathrm{mg} / \mathrm{dL}$ ( $8,52 \mathrm{mmol} / \mathrm{L})$

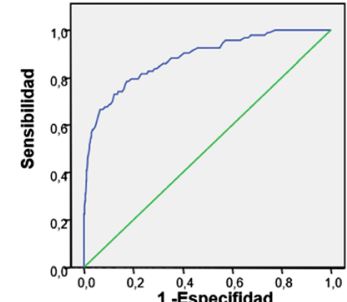

Punto de corte: $140,5 \mathrm{mg} / \mathrm{dL}(7,8 \mathrm{mmol} / \mathrm{L})$

$\mathbf{D}$

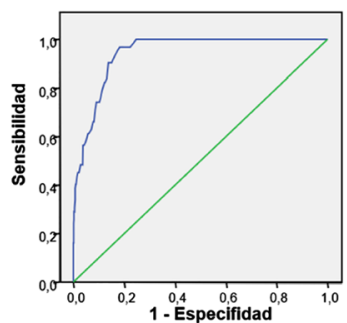

Punto de corte: $145,5 \mathrm{mg} / \mathrm{dL}(8,08 \mathrm{mmo} / \mathrm{L})$

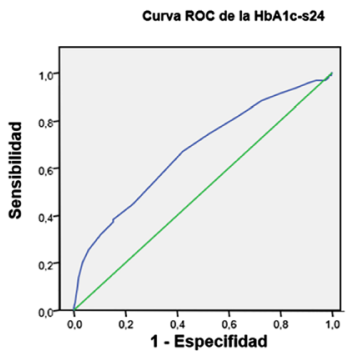

Punto de corte: $29 \mathrm{mmol} / \mathrm{mL}(4.85 \%)$

Curva ROC de la HbA1C-s24

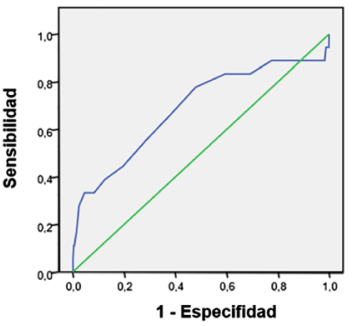

Punto de corte: $28 \mathrm{mmol} / \mathrm{mL}(4,75 \%)$ Curva ROC de la HbA1C-s13

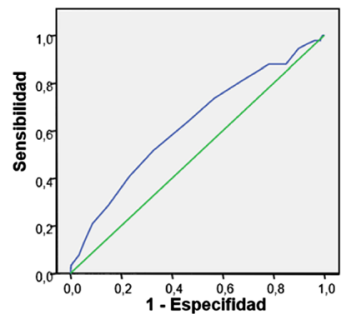

Punto de corle: $33 \mathrm{mmol} / \mathrm{mL}(5,15 \%)$ Curva ROC de la HbA1C-s24

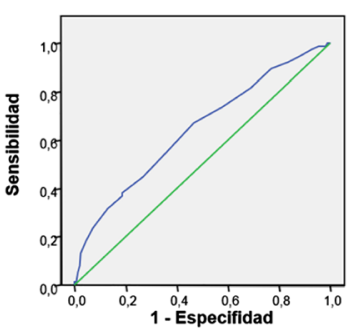

Punto de corte: $29 \mathrm{mmol} / \mathrm{mL}(\mathbf{4}, 85 \%)$

Figura 1: Curvas ROC que muestran la sensibilidad y especifidad del $\mathrm{TOS}^{\mathrm{a}}$ y la $\mathrm{HbA}_{1 \mathrm{c}}{ }^{\mathrm{b}}$ para el cribado de $\mathrm{DMG}^{\mathrm{c}}$.

(A) Sensibilidad y especifidad del TOS-s24d y la HbA1c-s24e en la población total. (B) Sensibilidad y especifidad del TOS-s24d y HbA1c-s24e en el grupo sin factores de riesgo. (C) Sensibilidad y especifidad del TOS-s13f y la HbA1c-s13g en el grupo con factores de riesgo. (D) Sensibilidad y especifidad del TOS-s24 y la HbA1c-s24 en el grupo con factores de riesgo. TOS ${ }^{\mathrm{a}}$, test de O'Sullivan; $\mathrm{HbA}_{1 \mathrm{c}}{ }^{\mathrm{b}}$, prueba de la hemoglobina glicosilada; $\mathrm{DMG}^{\mathrm{c}}$, diabetes mellitus gestacional; TOS-s2 $4^{\mathrm{d}}$, test de 0 'Sullivan en la semana $24^{\mathrm{a}}$; $\mathrm{HbA}_{1 c^{-}}$ s2 $4^{\mathrm{e}}$, prueba de la hemoglobina glicosilada en la semana $24^{\mathrm{a}}$; TOS-s13 ${ }^{\mathrm{f}}$, test de $0^{\prime}$ Sullivan en la semana $13^{\mathrm{a}} ; \mathrm{HbA}_{1 c^{-}} \mathrm{s} 13^{\mathrm{g}}$, prueba de la hemoglobina glicosilada en la semana $13^{\mathrm{a}}$.

\section{Análisis de la población con factores de riesgo}

Un total de 953 participantes presentaban algún factor de riesgo. La prevalencia de la DMG en esta población fue del $10 \%$. Los valores de TOS-s13 y $\mathrm{HbA}_{1 \mathrm{c}} \mathrm{s} 13$, GCT-s24, y $\mathrm{HbA}_{1 \mathrm{c}} \mathrm{s} 24$ fueron significativamente superiores en las participantes que desarrollaron DMG. No se observaron diferencias en cuanto a edad. Del mismo modo, las participantes que desarrollaron DMG con frecuencia presentaban un IMC $>30 \mathrm{~kg} / \mathrm{m}^{2}$ y habían desarrollado DMG anteriormente. No hubo diferencias estadísticamente significativas con respecto a la edad $>35$ años, la macrosomía y los antecedentes familiares (Tablas 2 and 3 ).

La regresión logística tanto en la semana $13^{\mathrm{a}}$ como en la semana $24^{\mathrm{a}}$ no mostró un buen rendimiento diagnóstico, ya que, aunque el análisis clasificó correctamente al 98,9\% de las participantes, solo identificó correctamente el 52\% de los casos de DMG.

En cuanto a la precisión diagnóstica en la semana $13^{\mathrm{a}}$, el TOS mostró un AUC de 0,882, siendo el mejor punto de corte $140,5 \mathrm{mg} / \mathrm{dL}$ (7,8 mmol/L) (Sensibilidad: 73,1\%; Especifidad: 87.7\%; VPP: 39,3\%; VPN: 96,75\%). La $\mathrm{HbA}_{1 \mathrm{c}}$ mostró un AUC de 0,624, siendo el mejor punto de corte $33 \mathrm{mmol} / \mathrm{mol}$ (5,15\%) (Sensibilidad 51,6\%; Especifidad: 67,3\%; VPP: 14,74\%; VPN: 92,65\%) (Figura 1C, Tabla 4). Con respecto a los umbrales extremos, el valor $26 \mathrm{mmol} /$ mol (4,55\%) mostró una sensibilidad del 94.5\% para descartar DMG, mientras que el valor $39 \mathrm{mmol} / \mathrm{mol}(5,75 \%)$ mostró una especifidad del 98,2\% para diagnosticar DMG.

En cuanto a la precisión diagnóstica en la semana $24^{\mathrm{a}}$, el TOS mostró un AUC de 0,944 siendo el mejor punto de corte 145,5 mg/dL (8,08 mmol/L) (Sensibilidad: 96,8\%; Especifidad: 82,7\%; VPP: 27,90\%; VPN: 99,71\%). La $\mathrm{HbA}_{1 \mathrm{c}}$ mostró un AUC de 0,642, siendo el mejor punto de corte $29 \mathrm{mmol} / \mathrm{mol}$ (4,85\%) (Sensibilidad: 67,1\%; Especifidad: 53,3\%; VPP: 11,69\%; VPN: 94,62\%) (Figura 1D, Tabla 4). Con respecto a los umbrales extremos, el valor $24 \mathrm{mmol} /$ mol (4,35\%) mostró una sensibilidad del 94,7\% para descartar DMG, mientras que el valor $39 \mathrm{mmol} / \mathrm{mol}$ (5,75\%) mostró una especifidad del 98,7\% para diagnosticar DMG.

\section{Validez diagnóstica de los modelos propuestos con datos reales}

Con los datos obtenidos, se desarrollaron dos métodos para la detección de DMG: (1) aumentar el punto de corte del TOS con objeto de reducir el número de gestantes que se 
Tabla 4: Estadísticos de precisión diagnóstica para cada estrategia.

\begin{tabular}{|c|c|c|c|c|c|c|}
\hline Población & Prueba & $\mathbf{S b}^{\mathrm{a}}$ & $\mathbf{S p}^{\mathrm{b}}$ & $\mathbf{P P V}^{\mathrm{c}}$ & $\mathbf{N P V}^{\mathbf{d}}$ & $\operatorname{AUC}^{e}\left(95 \% \mathrm{Cl}^{\mathrm{f}}\right)$ \\
\hline \multirow[t]{3}{*}{ Todas las gestantes } & TOS-s $24^{\mathrm{g}} \geq 140 \mathrm{mg} / \mathrm{dL}(\geq 7,8 \mathrm{mmol} / \mathrm{L})$ & 98,8 & 79,9 & 60 & 99,91 & $0,953(0,938-0,968)$ \\
\hline & TOS-s24>145,4 mg/dL (>8,07 mmol/L) & 95,1 & 85,7 & 22,19 & 99,75 & $0,953(0,938-0,968)$ \\
\hline & 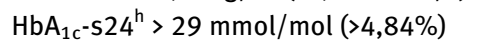 & 67 & 57,8 & 7,6 & 97,12 & $0,672(0,612-0,731)$ \\
\hline \multirow[t]{4}{*}{ Gestantes sin factores de riesgo } & TOS-s24 $\geq 140 \mathrm{mg} / \mathrm{dL}(\geq 7,8 \mathrm{mmol} / \mathrm{L})$ & 94,7 & 83,7 & 72 & 99,88 & $0,953(0,915-0,992)$ \\
\hline & TOS-s24>153,4 mg/dL (>8,51 mmol/L) & 89,5 & 93,6 & 20,48 & 99,79 & $0,953(0,915-0,992)$ \\
\hline & $\mathrm{HbA}_{1 \mathrm{c}}-\mathrm{s} 24>28 \mathrm{mmol} / \mathrm{mol}(>4,74 \%)$ & 77,8 & 52,1 & 2,87 & 99,22 & $0,688(0,541-0,834)$ \\
\hline & Algoritmo combinado s $24^{\mathrm{i}}$ & 77,8 & 95,2 & 22,95 & 99,57 & \\
\hline \multirow[t]{8}{*}{ Gestantes con factores de riesgo } & TOS-s13 $\geq 140 \mathrm{mg} / \mathrm{dL}(\geq 7,8 \mathrm{mmol} / \mathrm{L})$ & 73,1 & 87,0 & 49 & 96,68 & $0,882(0,843-0,921)$ \\
\hline & TOS-s13 ${ }^{\mathrm{i}}>140,4 \mathrm{mg} / \mathrm{dL}(>7,79 \mathrm{mmol} / \mathrm{L})$ & 73,1 & 87,7 & 39,3 & 96,75 & $0,882(0,843-0,921)$ \\
\hline & $\mathrm{HbA}_{1 \mathrm{c}}-\mathrm{S} 13^{\mathrm{k}}>33 \mathrm{mmol} / \mathrm{mol}(>5,14 \%)$ & 51,6 & 67,3 & 14,74 & 92,65 & $0,624(0,562-0,686)$ \\
\hline & Algoritmo combinado $\mathrm{s} 13^{\prime}$ & 67,8 & 89,1 & 40,39 & 96,21 & \\
\hline & TOS-s24 $\geq 140 \mathrm{mg} / \mathrm{dL}(\geq 7,8 \mathrm{mmol} / \mathrm{L})$ & 100 & 75,3 & 60 & 100 & $0,944(0,925-0,962)$ \\
\hline & TOS-s24>145,4 mg/dL (>8,07 mmol/L) & 96,8 & 82,7 & 27,9 & 99,71 & $0,944(0,925-0,962)$ \\
\hline & $\mathrm{HbA}_{1 \mathrm{c}}-\mathrm{s} 24>29 \mathrm{mmol} / \mathrm{mol}(>4,84 \%)$ & 67,1 & 53,3 & 11,69 & 94,62 & $0,642(0,575-0,709)$ \\
\hline & Algoritmo combinado s24 & 90 & 83,4 & 28,42 & 99,12 & \\
\hline
\end{tabular}

$\mathrm{Sb}^{\mathrm{a}}$, sensibilidad; $\mathrm{Sp}^{\mathrm{b}}$, especifidad; VPP ${ }^{\mathrm{c}}$, valor predictivo positivo; VPN ${ }^{\mathrm{d}}$, Valor predictivo negativo; AUC ${ }^{\mathrm{e}}$, Área bajo la curva; IC $\mathrm{C}^{\mathrm{f}}$, intervalo de confianza; TOS-s $24^{\mathrm{g}}$, test de O'Sullivan en la semana $24^{\mathrm{a}}$; $\mathrm{HbA}_{1 \mathrm{c}}-\mathrm{s} 24^{\mathrm{h}}$, prueba de la hemoglobina glicosilada en la semana $24^{\mathrm{a}}$; Algoritmo

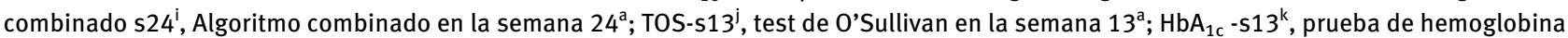
glicosilada en la semana $13^{\mathrm{a}}$. Algoritmo combinado $\mathrm{s} 13^{\mathrm{l}}$, Algoritmo combinado en la semana $13^{\mathrm{a}}$.

tienen que someter a la SOG; (2) emplear un algoritmo que combine un punto de corte de $\mathrm{HbA}_{1 \mathrm{c}}$ con buena sensibilidad para descartar DMG, seguido de un SOG con un punto de corte mayor.

\section{Gestantes $\sin$ factores de riesgo $(n=1054)$}

De acuerdo con la estrategia estándar, todas las gestantes de bajo riesgo tuvieron que someterse al TOS, de las que 187 precisaron una SOG. La prevalencia de DMG en la población con niveles de glucosa en plasma $<140 \mathrm{mg} / \mathrm{dL}$ $(<7,77 \mathrm{mmol} / \mathrm{L})(\mathrm{n}=866)$ fue del 0,1\% (un caso).

\section{Protocolo estándar mejorado (aumentar el punto de corte del TOS a153.5) (Figura 2A)}

Con este valor optimizado, solo se tendría que haber realizado la prueba a 83 gestantes. La prevalencia de DMG en la población con niveles de glucosa en plasma $<153 \mathrm{mg} / \mathrm{dL}$ $(<8,49 \mathrm{mmol} / \mathrm{L})(\mathrm{n}=971)$ fue del $0,2 \%$ (dos casos). Los parámetros estadísticos de este modelo se muestran en la Tabla 4.

\section{Algoritmo combinado (Figura 2A)}

Teóricamente, si se hubiera aplicado un algoritmo con un umbral de $\mathrm{HbA}_{1 \mathrm{c}}$ de $25 \mathrm{mmol} / \mathrm{mol}$ (4,45\%) para descartar DMG, habría que haber realizado el TOS a 782 mujeres. Posteriormente, aquellas con un TOS $>153,4(\mathrm{n}=61)$ precisarían la SOG (49 observaciones perdidas de las 1054). En esta cohorte de gestantes, y teniendo en cuenta el diagnóstico final, los parámetros estadísticos del algoritmo fueron: Sensibilidad: 77,8\%; Especifidad: 95,2\%; VPP: 22,95\%, y VPN: 99,57\% (Tabla 4).

\section{Gestantes con factores de riesgo $(n=953)$ en la semana $13^{a}$}

Con el protocolo clásico, se hubiera realizado el TOS a todas las gestantes con factores de riesgo en la semana $13^{\mathrm{a}}$ $(\mathrm{n}=953)$; posteriormente, aplicando el punto de corte estándar (140 mg/dL; 7,77 mmol/L), un total de 188 mujeres precisarían una SOG.

\section{Protocolo estándar mejorado (aumentar el punto de corte del TOS a 140,5) (Figura 2B)}

Con el nuevo punto de corte, solo se tendría que haber realizado la SOG a 182 mujeres. La prevalencia de la DMG en la población con valores inferiores al mismo fue del $3,2 \%$, por lo que 25 casos no habrían sido diagnosticados. Los parámetros estadísticos de este modelo se muestran en la Tabla 4.

\section{Algoritmo combinado (Figura 2B)}

Un punto de corte para la $\mathrm{HbA}_{1 \mathrm{c}}$ de $26 \mathrm{mmol} / \mathrm{mol}(4,55 \%)$ permitiría descartar la DMG con una sensibilidad del 94,5\%. No obstante, en condiciones reales de uso, aunque nos permitiría descartar DMG en 89 mujeres, cinco se 

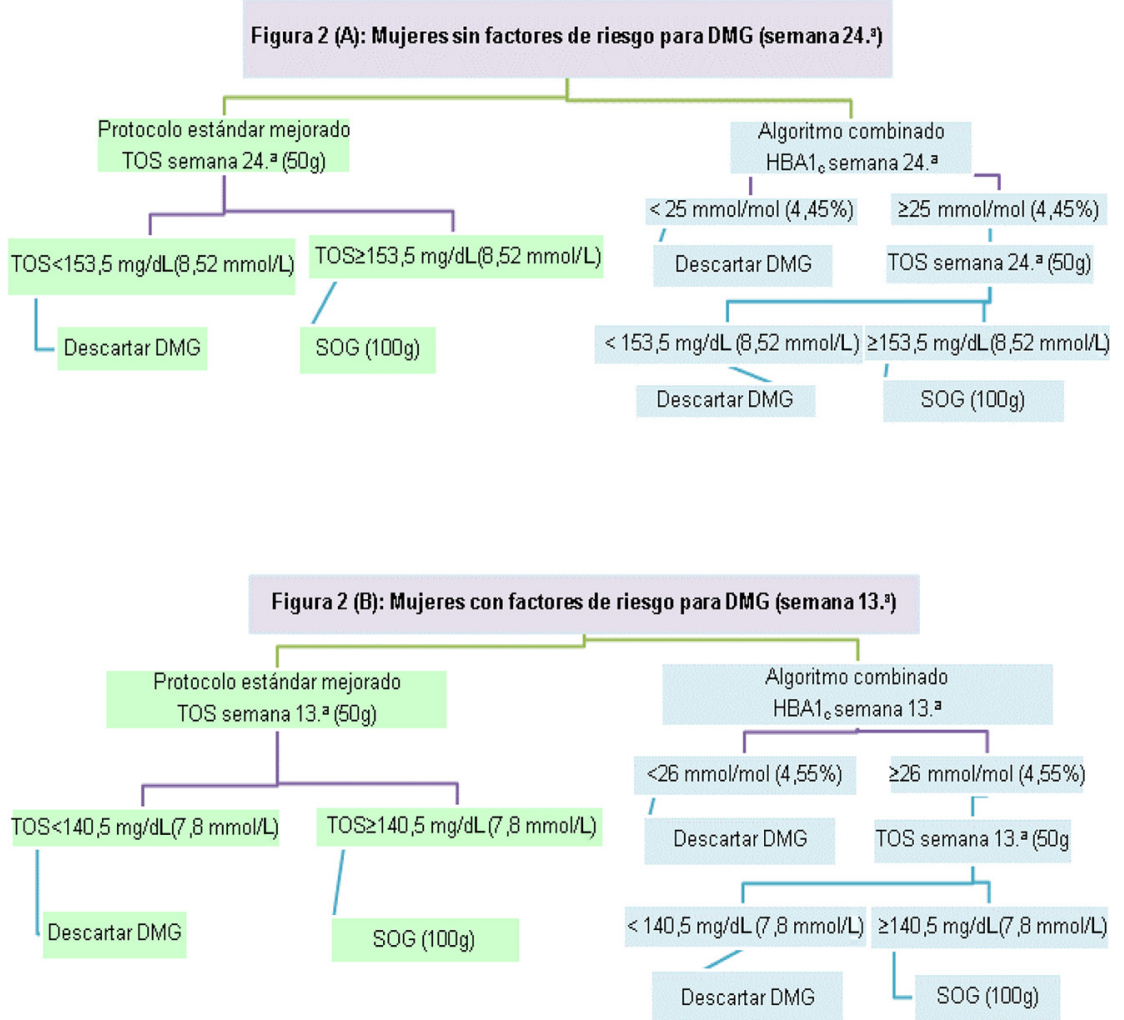

Figura 2: Flujo de trabajo de las dos estrategias para el cribado de DMG. DMG, diabetes mellitus gestacional; TOS-13 ${ }^{\mathrm{a}}$ semana, test de 0'Sullivan en la semana $13^{\mathrm{a}} ; \mathrm{HbA}_{1 c^{-}}-13^{\mathrm{a}}$ semana, prueba de la hemoglobina glicosilada en la semana $13^{\mathrm{a}}$; TOS- $24^{\mathrm{a}}$ semana, test de O'Sullivan en la semana $24^{\mathrm{a}} ; \mathrm{HbA}_{1 \mathrm{c}}-24^{\mathrm{a}}$ semana, prueba de la hemoglobina glicosilada en la semana $24^{\mathrm{a}}$. habrían quedado sin diagnosticar (prevalencia de la DMG del $5,6 \%$ en la población con un $\mathrm{HbA}_{1 \mathrm{c}}<26 \mathrm{mmol} / \mathrm{mol}$ $(4,55 \%))$. Posteriormente, aquellas con un $>140.4(n=151)$ precisarían una SOG (32 observaciones perdidas de 953). Teniendo en cuenta el diagnóstico final, los parámetros estadísticos de este algoritmo fueron: Sensibilidad: $67,8 \%$, Especifidad: 89,1\%, VPP: 40,39\%, VPN: 96,21\% (Tabla 4).

\section{Gestantes con factores de riesgo $(n=901)$ en la semana $24^{\mathrm{a}}$}

Con el método tradicional, en la semana $24^{\mathrm{a}}$, se hubieran realizado el TOS todas las participantes con factores de riesgo ( $\mathrm{n}=901$ ); posteriormente, aplicando el punto de corte estándar ( $140 \mathrm{mg} / \mathrm{dL} ; 7,77 \mathrm{mmol} / \mathrm{L})$, un total de 272 mujeres precisarían una SOG.

\section{Protocolo estándar mejorado (aumentar el punto de corte del TOS a 145.5) (Figura 2C)}

Un total de 215 mujeres presentaron un TOS >145,4; por lo que, teóricamente, precisarían una SOG. La prevalencia de DMG en esta población fue del 27,9\% (60 casos). En el grupo con TOS $<145,5$, la prevalencia de la DMG fue del 0,3\% (solo 2 casos). Los parámetros estadísticos de este modelo se muestran en la Tabla 4. 


\section{Algoritmo combinado (Figura 2C)}

Un punto de corte de $24 \mathrm{mmol} / \mathrm{mol}(4,35 \%)$ para $\mathrm{HbA}_{1 \mathrm{c}}$ permitiría descartar la DMG, con una sensibilidad del $94,7 \%$. Usando datos reales, este valor nos permitiría descartar la DMG en 100 mujeres, pero cuatro hubiesen quedado sin diagnosticar (prevalencia de la DMG del $4 \%$ en la población con un $\mathrm{HbA}_{1 \mathrm{c}}<24 \mathrm{mmol} / \mathrm{mol}(4,35 \%)$ ). Posteriormente, 801 mujeres se tendrían que haber sometido al TOS. De estas, 190 mostraron un resultado $\geq 145,5$ y deberían realizarse la SOG con $100 \mathrm{~g}$ ( 23 observaciones perdidas de 901). Teniendo en cuenta el diagnóstico final, los parámetros estadísticos de este algoritmo fueron: Sensibilidad: 90,0\%, Especifidad: 83,4\%, VPP: 28,42\%, VPN: $99,12 \%$.

\section{Método del punto de corte extremo para el diagnóstico temprano de DMG en gestantes con riesgo elevado}

Si empleáramos un punto de corte con una especifidad teórica para diagnosticar DMG del 98,2\%, $39 \mathrm{mmol} / \mathrm{mol}$ $(5,75 \%)$ en nuestra población, se habrían diagnosticado 20 casos, aunque la prevalencia real de la DMG en este subgrupo fue solo del 25\% (cinco casos).

\section{Discusión}

Este estudio muestra que el procedimiento estándar (test de O'Sullivan) mejorado con puntos de corte más altos, ofrece la mejor validez diagnóstica para la DMG. La $\mathrm{HbA}_{1 \mathrm{c}}$ podría ser útil con este fin, aunque tiene una validez diagnóstica inferior a la del método de referencia. Aunque la prueba de $\mathrm{HbA}_{1 \mathrm{c}}$ obtuvo un AUC adecuado, esta fue muy inferior a la obtenida con el TOS en todos los grupos estudiados. Esto podría deberse en gran medida a la baja sensibilidad de la $\mathrm{HbA}_{1 \mathrm{c}}$ observada consistentemente en los grupos. La precisión diagnóstica de la prueba de $\mathrm{HbA}_{1 \mathrm{c}}$ para la DMG ha sido analizada recientemente [21-24]. El valor del AUC en estos estudios oscila entre 0,62 y 0,72 . En los mismos, se concluye que la $\mathrm{HbA}_{1 \mathrm{c}}$ no puede sustituir a las pruebas basadas en la sobrecarga de glucosa, aunque podría ser de utilidad como prueba de cribado [23, 24] o para identificar a las embarazadas con un riesgo elevado de desarrollar DMG [21]. Además, el papel de la $\mathrm{HbA}_{1 \mathrm{c}}$ en el diagnóstico temprano de la DMG en las mujeres con alto riesgo ha sido analizado en diversos estudios [26-28]. Con este propósito, en estos trabajos se han empleado puntos de corte extremos que, al maximizar la especifidad, permitirian diagnosticar la DMG en aquellas mujeres que mostraran valores superiores a los mismos. En una revisión sistemática, Kattini et al. [28] concluyeron que un valor límite de entre el 5,7\% y el 6,4\% identifica a aquellas pacientes que acabarán desarrollando DMG. En nuestro estudio, al aplicar el método de los puntos de corte extremos, obtuvimos varios umbrales de $\mathrm{HbA}_{1 \mathrm{c}}$, tanto para la semana $13^{\mathrm{a}}$ como para la semana $24^{\mathrm{a}}$ de gestación. Teóricamente, estos umbrales podrían diagnosticar o descartar la DMG, maximizando la especifidad o la sensibilidad. El mejor punto de corte para el diagnóstico temprano de la DMG en las pacientes con alto riesgo fue 5,75\% (39 mmol/L). Sin embargo, al aplicar el modelo a nuestra población, solo el $25 \%$ de los casos con valores superiores a esta cifra desarrollaron DMG. Estos resultados coinciden con los obtenidos por Fong et al. [20], en los que el 27,3\% de las embarazadas con un valor de $\mathrm{HbA}_{1 \mathrm{c}}$ superior al 5,7\%, evaluadas antes de la semana $20^{\mathrm{a}}$ de gestación desarrollaron DMG. Además, coincide con los resultados de Punnose et al. [29] y Walker et al. [30], quienes concluyeron que la prueba de $\mathrm{HbA}_{1 \mathrm{c}}$ ni es superior a las pruebas basadas en la sobrecarga de glucosa, ni resulta rentable. Por lo tanto, la baja AUC de $\mathrm{HbA}_{1 \mathrm{c}}$ en nuestro estudio, así como en otros $[26,29]$, sugiere que estos puntos de corte extremos podrían no ser adecuados.

De acuerdo con otros autores [31-33], se propuso un algoritmo combinado. Sin embargo, este método difiere del resto en que se trata de una estrategia en tres pasos donde: (a) en la primera cita con el obstetra, se clasifica a las gestantes (semana $12^{\mathrm{a}}$ ) como con bajo riesgo o con riesgo elevado, según la presencia o no de factores de riesgo conocidos; (b) se realiza la $\mathrm{HbA}_{1 \mathrm{c}}$ en la semana $13^{\mathrm{a}}$ y/o en la semana $24^{a}$, según proceda, con el objeto de descartar la DMG; (c) para aquellos casos en los que haya que realizar el TOS, se eleva el umbral de la prueba tanto para las gestantes con bajo riesgo como para aquellas con un riesgo elevado, con el fin de evitar la realización innecesaria de la SOG. Sin embargo, los algoritmos resultantes mostraron un rendimiento diagnóstico inferior que el protocolo estándar mejorado (Tabla 4).

Al elaborar este artículo, la pandemia de COVID-19 se ha convertido en el mayor problema de salud pública de las últimas décadas. Con el fin de evitar la posible exposición de las gestantes al virus, autoridades sanitarias y sociedades científicas, han propuesto nuevos protocolos para el diagnóstico de DMG basados en la $H b A 1_{c}$ y la glucosa en plasma en ayunas o aleatoria [34-37]. La eficacia de estos métodos a para detectar DMG y sus posibles complicaciones en el embarazo se han estudiado exhaustivamente a partir de datos del "Estudio sobre la Hiperglucemia y los Resultados Adversos del Embarazo" (HAPO) [38]. Este estudio muestra que los métodos basados en la prueba de $\mathrm{HbA}_{1 \mathrm{c}}$ y/o de glucosa en plasma 
en ayunas [34, 35] están asociados con un aumento en el número de gestantes con DMG no diagnosticada, con indices significativamente mayores de complicaciones en el embarazo. Sin embargo, el método basado en los niveles de glucosa en ayunas, seguido de una prueba de SOG selectiva [36], aunque asociado con un mayor número de DMG no diagnosticada, no está unido a una mayor incidencia de resultados adversos. Por todo ello, los autores recomiendan que se evalúen los riesgos y beneficios de cada propuesta, en el contexto actual de pandemia.

En este estudio se demuestra que se debería reconsiderar en todos los grupos el punto de corte estándar del TOS (140 mg/dL: 7,77 mmol/L). Para las gestantes de bajo riesgo, el nuevo valor es especialmente relevante (153,5 mg/dL: $8,52 \mathrm{mmol} / \mathrm{L})$. Aumentar la especifidad podría implicar menor sensibilidad de la prueba. No obstante, este punto de corte mostró una sensibilidad del $89,5 \%$ y, en la práctica real, los casos sin diagnosticar aumentarían ligeramente del $0,1 \%$ al $0,2 \%$. En el contexto de bajos índices de prevalencia comunitarios, como es el caso de nuestra cohorte, se ha propuesto ajustar el punto de corte del TOS a $145 \mathrm{mg} / \mathrm{dL}(8,05 \mathrm{mmol} / \mathrm{L})$ en embarazos gemelares para evitar falsos positivos de DMG [39]. Además, la estrategia mejorada podría ser costo-eficiente. En la población de bajo riesgo, se podrían haber evitado el 55,6\% de los TOS. En la población de alto riesgo en la semana $13^{\mathrm{a}}$ solo se habría evitado realizar el 3,2\% de las SOG. Sin embargo, en la semana $24^{\mathrm{a}}$, la ventaja fue evidente, pudiéndose haber evitado el 21\% de las SOG.

\section{Puntos fuertes y limitaciones del estudio}

Este estudio se ha realizado con una de las mayores cohortes publicadas hasta la fecha. Su principal limitación radica en que en España se aplica un método diferente al recomendado por la ADA o la OMS para diagnosticar DMG, lo que limita en parte la extrapolación de los resultados obtenidos. Otras limitaciones son que el presente estudio es unicéntrico, con una prevalencia de DMG del 5,7\% y las gestaciones múltiples no se excluyeron (prevalencia $<1,5 \%$ ). Aunque hubo un $9,7 \%$ de pérdidas durante el seguimiento, es un porcentaje aceptable que no pone en riesgo la validez de los resultados obtenidos.

\section{Conclusiones}

Este estudio muestra que se puede optimizar el cribado de DMG aplicando nuevos puntos de corte para el O’Sullivan.
Además, se muestra que, en términos de precisión diagnóstica, la $\mathrm{HbA}_{1 \mathrm{c}}$ por sí misma o en un algoritmo combinado, es inferior a la del método mejorado del O'Sullivan. Por otra parte, el método del punto de corte extremo para el diagnóstico temprano de la DMG en la población de alto riesgo basado en la $\mathrm{HbA}_{1 \mathrm{c}}$ resulta menos eficiente que la estrategia estándar o la mejorada propuesta en este estudio.

Es necesario realizar más estudios sobre los puntos de corte del test de O'Sullivan para poder aplicar cambios reales al protocolo de cribado de DMG.

Agradecimientos: Los autores agradecen al Instituto Nacional Carlos III por financiar el presente estudio.

Financiación de la investigación: Este estudio fue financiado mediante una beca del Instituto Nacional Carlos III (ISCIII) (PI11 01064).

Contribución de los autores: Todos los autores aceptan la responsabilidad sobre la totalidad del contenido del manuscrito enviado, habiendo todos aprobado su presentación.

Conflicto de intereses: Los autores declaran no tener ningún conflicto de intereses.

Aprobación ética: La investigación se realizó de conformidad con la legislación nacional, las políticas institucionales, y de acuerdo con los principios de la Declaración de Helsinki. Así mismo, este estudio fue aprobado por el Comité de Ética del Hospital Reina Sofía, Córdoba (España).

\section{Referencias}

1. International Association of Diabetes and Pregnancy Study Groups Consensus Panel. International association of diabetes and pregnancy study groups recommendations on the diagnosis and classification of hyperglycemia in pregnancy. Diabetes Care 2010;33:676-82.

2. World Health Organization. Diagnostic criteria and classification of hyperglycemia first detected in pregnancy; 2013. WHO/NMH/ MND/13.2. Available from: http://www.who.int/diabetes/ publications/Hyperglycaemia_In_Pregnancy/en/.

3. American Diabetes Association. Standards of medical care in diabetes-2019 Abridged for primary care providers. Clin Diabetes 2019;37:11-34.

4. Daly N, Flynn I, Carroll C, Farren M, McKeating A, Turner MJ. A national survey of preanalytical handling of oral glucose tolerance tests in pregnancy. Clin Chem Lab Med 2016;54:221-3.

5. Mack LR, Tomich PG. Gestational Diabetes diagnosis, classification, and clinical care. Obstet Gynecol Clin N Am 2017;44:207-17.

6. Durán A, Calle AL. Diagnostic criteria for gestational diabetes: the debate goes on. Endocrinol Nutr 2015;62:207-9.

7. Behboudi-Gandevani S, Amiri M, Bidhendi Yarandi R, Ramezani Tehrani F. The impact of diagnostic criteria for gestational diabetes on its prevalence: a systematic review and meta-analysis. Diabetol Metab Syndrome 2019;11:1-18. 
8. Buckley BS, Harreiter J, Damm P, Corcoy R, Chico A, Simmons D, et al. Gestational Diabetes in Europe: prevalence, current screening practice and barriers to screening. A review. Diabet Med 2012;29:844-54.

9. Ricart W, López J, Mozas J, Pericot A, Sancho MA, González N, et al. Potential impact of American Diabetes Association (2000) criteria for diagnosis of gestational diabetes in Spain. Diabetologia 2005;48:1135-41.

10. Grupo Español de Diabetes y Embarazo (GEDE). Asistencia a la gestante con diabetes mellitus y embarazo. Guía de práctica clínica actualizada en 2014. Av Diabetol 2015;31:45-59.

11. National Diabetes Data Group. Classification and diagnosis of diabetes mellitus and other categories of glucose intolerance. Diabetes 1979;18:1039-57.

12. Daly N, Stapleton M, O’Kelly R, Kinsley B, Daly S, Turner MJ. The role of preanalytical glycolysis in the diagnosis of gestational diabetes in obese women. Am J Obstet Gynecol 2015;213:84.

13. van den Berg SA, van Thiel SW, Thelen MH. Updating pregnancy diabetes guidelines: is (y)our laboratory ready? Clin Chem Lab Med 2016;54:225-7.

14. Potter JM, Hickman PE, Oakman C, Woods C, Nolan CJ. Strict preanalytical oral glucose tolerance test blood sample handling is essential for diagnosing gestational diabetes mellitus. Diabetes Care 2020;43:1438-41.

15. Lachmann EH, Fox RA, Dennison RA, Usher-Smith JA, Meek CL, Aiken CE. Barriers to completing oral glucose tolerance testing in women at risk of gestational diabetes. Diabet Med 2020;6: 1482-9.

16. García-Del-Pino I, Bauça JM, Gómez C, Caballero A, Llopis MA, Ibarz $M$, et al. Preanalytical issues related to routine and diagnostic glucose tests: results from a survey in Spain. Biochem Med (Zagreb) 2020;30:010704.

17. International Expert Committee. International Expert Committee report on the role of the $\mathrm{A} 1 \mathrm{C}$ assay in the diagnosis of diabetes. Diabetes Care 2009;32:1327-34.

18. Santos-Rey K, Fernández-Riejos P, Mateo J, SánchezMargalet V, Goberna R. Glycated hemoglobin versus the oral glucose tolerance test for the exclusion impaired glucose tolerance in high-risk individuals. Clin Chem Lab Med 2010; 48:1719-22.

19. Manley SE, Sikaris KH, Lu ZX, Nightingale PG, Stratton IM, Round RA, et al. Validation of an algorithm combining haemoglobin A1C and fasting plasma glucose for diagnosis of diabetes mellitus in UK and Australian populations. Diabet Med 2009;26:115-21.

20. Fong A, Serra AE, Gabby L, Wing DA, Berkowitz KM. Use of hemoglobin A1c as an early predictor of Gestational Diabetes. Am J Obstet Gynecol 2014;211:641-3.

21. Benaiges D, Flores-Le Roux JA, Marcelo I, Mañé L, Rodríguez M, Navarro X, et al. Is first-trimester $\mathrm{HbA}_{1 \mathrm{c}}$ useful in the diagnosis of gestational diabetes? Diabetes Res Clin Pract 2017;133: 85-91.

22. Rayis DA, Ahmed ABA, Sharif ME, ElSouli A, Adam I. Reliability of glycosylated hemoglobin in the diagnosis of gestational diabetes mellitus. J Clin Lab Anal 2020;2:e23435.

23. Maesa JM, Fernandez-Riejos P, Gonzalez-Rodriguez C, SanchezMargalet V. Screening for gestational diabetes mellitus by measuring glycated hemoglobin can reduce the use of the glucose challenge test. Ann Lab Med 2019;39:524-9.

24. Siricharoenthai $P$, Phupong V. Diagnostic accuracy of $\mathrm{HbA}_{1 \mathrm{c}}$ in detecting gestational diabetes mellitus. J Matern Fetal Neonatal Med 2019;28:1-11.

25. Renz PB, Chume FC, Timm JRT, Pimentel AL, Camargo JL. Diagnostic accuracy of glycated hemoglobin for gestational diabetes mellitus: a systematic review and meta-analysis. Clin Chem Lab Med 2019;57:1435-49.

26. Hughes RC, Moore MP, Gullam JE, Mohamed K, Rowan J. An Early $\left(\mathrm{HbA}_{1 \mathrm{c}} \geq 5.9 \%\right)(41 \mathrm{mmol} / \mathrm{ml})$ is optimal for detecting diabetes and identifies women at increased risk of adverse pregnancy outcomes. Diabetes Care 2014;37:2953-9.

27. Battarbee AB, Grant JH, Vladutiu CJ, Menard MK, Clark M, Manuck TA, et al. Hemoglobin A1c and early gestational diabetes. J Womens Health (Larchmt) 2020;15:1559-63.

28. Kattini R, Hummelen R, Kelly L. Early gestational diabetes mellitus screening with glycated hemoglobin: a systematic review. J Obstet Gynaecol Can 2020;5:S1701-2163.

29. Punnose J, Malhotra RK, Sukhija K, Mathew A, Sharma A, Choudhary N. Glycated haemoglobin in the first trimester: a predictor of gestational diabetes mellitus in pregnant Asian Indian women. Diabetes Res Clin Pract 2020;159:107953.

30. Walker AR, Caughey AB. Positivity thresholds of $\mathrm{HbA}_{1 \mathrm{c}}$ assay as a screening test for diabetes mellitus in the first trimester in highrisk populations. J Matern Fetal Neonatal Med 2020;9:1-5.

31. Verhaeghe J, Van Herck E, Benhalima K, Mathieu C. Glycated hemoglobin in pregnancies at risk for gestational diabetes. Eur J Obstet Gynecol Reprod Biol 2012;161:157-62.

32. Rajput R, Yadav $Y$, Rajput $M$, Nanda S. Utility of $\left(\mathrm{HbA}_{1 \mathrm{c}}\right)$ for the diagnosis of gestational diabetes. Diabetes Res Clin Pract 2012; 98:104-7.

33. Aldasouqi SA, Gossain VV. Update on diabetes diagnosis: a historical review of the dilemma of the diagnostic utility of glycohemoglobin A1C and a proposal for a combined glucose-A1C diagnostic method. Ann Saudi Med 2012;32:229-35.

34. Royal College of Obstetricians and Gynaecologists. Guidance for maternal medicine services in the evolving coronavirus (COVID-19) pandemic; 2020. https://www.rcog.org.uk/ globalassets/documents/guidelines/2020-07-10-guidance-formaternal-medicine.pdf [Accessed 4 Oct 2020].

35. Yamamoto JMDL, Reig DS, Berger H. Urgent update - temporary alternative screening strategy for gestational diabetes screening during the COVID-19 pandemic; 2020. https://els-jbs-prod-cdn. jbs.elsevierhealth.com/pb/assets/raw/Health\%20Advance/ journals/jcjd/JCJD_COVID_guidelines_020420-1585856697530. pdf [Accessed 4 Oct 2020].

36. Australasian Diabetes in Pregnancy Society. Diagnostic testing for gestational diabetes mellitus (GDM) during the COVID 19 pandemic. Antenatal and postnatal testing advice; 2020. https://www.adips. org/documents/COVID19-WITHQLDGUIDELINES0704201150 ADIPSADSADEADAupdated.pdf [Accessed 4 Oct 2020].

37. Codina M, Corcoy R, Goya MM. Actualización del diagnóstico de hiperglucemia gestacional durante la pandemia COVID-19. Update of the hyperglycemia Gestational diagnosis during the COVID-19 pandemic. Endocrinologíaa, diabetes y nutrición 2020; 67:545-52. 
38. McIntyre HD, Gibbons KS, Ma RCW, Tam WH, Sacks DA, Lowe J, et al. Testing for gestational diabetes during the COVID-19 pandemic. An evaluation of proposed protocols for the United Kingdom, Canada and Australia. Diabetes Res Clin Pract 2020;167:108353.

39. Jung YJ, Kwon JY, Cho HY, Park YW, Kim YH. Comparison of the performance of screening test for gestational diabetes in singleton versus twin pregnancies. Obstet Gynecol Sci 2015;58:439-45.
Material Suplementario: La versión en línea de este artículo ofrece material suplementario (https://doi.org/10.1515/almed-2020-0118).

Nota del artículo: El artículo original puede encontrarse aquí: https:// doi.org/10.1515/almed-2020-0072. 